\title{
10.21611/qirt.2016.a
}

\section{An Integrated Index for Breast Cancer Identification using Histogram of Oriented Gradient and Kernel Locality Preserving Projection Features Extracted from Thermograms}

\author{
E. Y. K. Nga, U. Raghavendra ${ }^{\mathrm{b}}$, U. Rajendra Acharya ${ }^{\mathrm{c}, \mathrm{d}, \mathrm{e}}$, Jen-Hong Tan ${ }^{\mathrm{c}}$, Anjan Gudigar ${ }^{\mathrm{e}}$ \\ ${ }^{a}$ School of Mechanical and Aerospace Engineering, College of Engineering, Nanyang Technological University, 50 \\ Nanyang Avenue, Singapore 639798. \\ ${ }^{b}$ Department of Instrumentation and Control Engineering, Manipal Institute of Technology, Manipal University, \\ Manipal, India 576104. \\ 'Department of Electronics and Computer Engineering, Ngee Ann Polytechnic, Singapore 599489. \\ ${ }^{d}$ Department of Biomedical Engineering, School of Science and Technology, SIM University, Singapore 599491. \\ ${ }^{e}$ Department of Biomedical Engineering, Faculty of Engineering, University of Malaya, Malaysia.
}

\begin{abstract}
Breast cancer is one of the prime causes of death in women worldwide. Thermography has shown a great potential in screening the breast cancer and overcomes the limitations of mammography. Moreover, interpretations of thermogram images are dependent on the specialists, which may lead to errors and uneven results. Preliminary screening method should detect the hazardous, destructive tumors effectively to improve the accuracy. The growth of malignant tumor can increase the internal temperature which can be captured by thermograms. Thus in this work, locally normalized Histogram of Oriented Gradients (HOG) based preliminary screening Computer Aided Diagnosis (CAD) tool is proposed. HOG is able to record the minute internal variations in thermograms. In order to reduce the dimensions of extracted HOG descriptors Kernel Locality Preserving Projection (KLPP) is used. The resulting KLPP features are then ranked to form an efficient classification model. Various machine learning algorithms are used to validate the proposed method. Our method shows a promising performance with an average accuracy, sensitivity, and specificity of $98 \%, 96.66 \%$ and $100 \%$ respectively. We have also developed a Breast Cancer Risk Index (BCRI) using significant KLPP features which can discriminate the two classes using a single integrated index. This can help the radiologists to discriminate the normal and malignant classes during screening to validate their findings.
\end{abstract}

Keywords: breast cancer; CAD; classification; features; HOG; thermography

\section{ACKNOWLEDGEMENT:}

The first author (Ng) acknowledges the SAF-NTU Academy and SAF's support for his attendance/participation to the conference.

\section{Brief CV:}

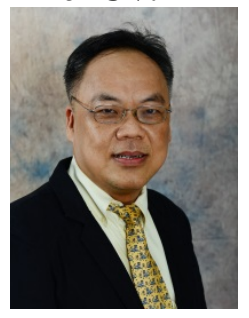

E.Y.K. Ng received his Ph.D. from Cambridge University, is an associate professor at Nanyang Technological University. He serves as Editor-in Chief for two SCIE indexed Journals. His research interests are thermal imaging, biomedical engineering, and computational fluid dynamics / heat transfer. He has published more than 265 ISI journal articles and 80 conference papers and 11 books. He has supervised more than 30 Master and PhD students and amassed over SGD\$5M worth of research funding from various organizations in the capacity of the principal investigator.

See URL at http://www.mae.ntu.edu.sg/aboutus/FacultyandStaff/Faculty/Pages/mykng.aspx 